\title{
Desarrollo forestal
} comunitario sustentable en la región norte de México y su desafío en el

\section{contexto de la globalización}

\section{Sustainable forest community development in northern Mexico and its challenge in the globalization context}

\author{
Concepción Luján Álvarez*, Jesús Miguel Olivas Garcíal, Hilda Guadalupe González Hernández², Susana Vázquez \\ Álvarez³, José Ciro Hernández Díaz y Humberto Luján Álvarez ${ }^{5}$
}

\begin{abstract}
1 Universidad Autónoma de Chihuahua. Facultad de 3 Consultora Forestal. Ciudad Delicias, Chihuahua. Ciencias Agrícolas y Forestales. Chihuahua. Mex jolivas@live.com.mx. hilda.gonzález@conafor.gob.mx.

3 Consultora Forestal. Ciudad Delicias, Chihuahua.
p76977@amail.com.
4 Universidad Juárez del Estado de Durango. Institu-
to de Silvicultura e Industria de la Madera. Durango,

Dgo. jciroh@ujed.mx

\author{
5 Secretaría de Educación Pública. Centro de Estudios \\ Tecnológicos y de Servicios \# 122. Chihuahua, Mex. \\ hlujan@prodigy.net.mx \\ * Autor para correspondencia: clujanl2@hotmail.com
}

\section{RESUMEN}

Los objetivos del estudio fueron analizar el desarrollo forestal comunitario sustentable en la región norte de México, en los estados de Chihuahua y Durango, considerando la organización comunitaria, flexibilidad, innovación, capacidad de respuesta, competitividad y colaboración, así como comercialización, ante la continua apertura de mercados. También, establecer una estrategia integral para impulsar el desarrollo forestal comunitario sustentable en ambos estados, como una región forestal conjunta-clúster forestal, en el contexto de la globalización económica. En el estudio fueron aplicadas encuestas a ejidos/comunidades, considerando la tipología de las cuatro categorías de productores forestales de Conafor, así como a informantes clave externos y además se incluyeron evidencias documentales. Los resultados muestran que ejidos y comunidades no son competitivos y tienen limitada organización; por esta razón, la estrategia integral propuesta incluye la integración horizontal y vertical de la cadena productiva, así como el empoderamiento de ejidos y participación comunitaria. Esta estrategia integral pretende fomentar la competitividad del desarrollo forestal comunitario sustentable en la globalización.

Palabras Clave: clúster forestal, competitividad forestal, comunidad forestal sustentable, estrategia forestal integral, ejidos forestales, globalización económica.

\section{ABSTRACT}

The objectives of this research were to analyze the sustainable community forestry in northern Mexico, in the states of Chihuahua and Durango, regarding the current status of communitarian organization, flexibility, responsiveness and innovation, competitiveness and collaboration, and marketing forest, at the continued opening of markets. Also, to establish an integral strategy to promote sustainable community forestry in both states, as a joint forest region-forest cluster, in the context of economic globalization. In the study, surveys were applied to ejidos/communities, considering Conafor's type of the four categories of forest producers, and to external key informants; also, documentary evidences were consulted in accordance with the objectives of the study. The results show that ejidos and communities are not competitive and that they have limited organization; for this reason, the proposed integral strategy includes the horizontal and vertical integration of the productive chain, empowerment, and community participation. The integral strategy aims to foster the competitiveness of sustainable community forestry development in the globalization.

KEYWORDS: forestry cluster, forestry competitiveness, sustainable community forestry, integral forestry strategy, forest ejido, economic globalization. 


\section{INTRODUCCIÓN}

En la globalización, con la apertura de mercados, hay nuevas oportunidades y desafíos para que las inversiones, el capital y las tecnologías aporten ventajas comparativas y competitivas para el desarrollo de la empresa o comunidad (Porter, 1990). En este contexto, la asociatividad de empresas, y en específico las empresas forestales comunitarias (EFC), continúa emergiendo en el mundo como una estrategia para avanzar en niveles de competitividad (Nolan, 2001; Gill, 2002; Cortave, 2003; Scherr, White y Kaimowitz, 2003), facilitando el "empoderamiento" comunitario para su mejor desarrollo (Redclift, 1987; Friedmann, 1992; Hirschman, 1993).

\section{Globalización económica y competitividad forestal}

Los bosques del mundo y el desarrollo del sector forestal están mostrando cambios sin precedente en los aspectos climáticos, socioeconómicos, políticos y biológicos. Por ello, es necesario establecer y operar principios amplios y promover cambios que permitan incrementar las capacidades adaptativas de los bosques y el sector forestal ante un futuro de incertidumbre (Messier et al., 2015).

Los procesos de apertura internacional e integración representan, en apariencia, un poderoso instrumento para la expansión del comercio y la inversión entre países (Smith y Cossio, 2008). A la vez, estudios han mostrado que existe una correlación positiva con significancia entre el nivel de sustentabilidad de las prácticas de administración estratégica y la efectividad en los mercados de las empresas (Witek-Crabb, 2012). Por ello, la fuerte competencia y la complejidad, prevaleciente en momentos en que la globalización tiene cada vez más auge e impacto, están marcando tendencias para que las organizaciones concentren sus esfuerzos en gestionar estratégicamente y de manera participativa sus capacidades para impulsar un desarrollo sustentable con visión de futuro (Trejo, 2013). Por ello, la experiencia de las organizaciones indica que el mejor camino para alcanzar la capacidad de competir es a través de la tecnología e innovación, factores clave de la competitividad (Carroz, 2005).
A nivel internacional, la relevancia de la competitividad en la producción forestal sustentable ha ido en aumento debido a los grandes retos que ha significado la globalización económica. En función de lo anterior, en el ámbito nacional se han encaminado esfuerzos a la búsqueda de vías institucionales, legales y financieras que permitan mejorar la competitividad del sector forestal y a la vez ser consistentes con los planteamientos del desarrollo sustentable establecido en la política forestal nacional. Sin embargo, la tarea de elevar el nivel de competitividad en el sector forestal ha demostrado ser un tema altamente complejo debido a que esta incluye muchos factores críticos asociados. Uno de ellos, es la aplicación de un proceso de descentralización en la toma de decisiones de manera participativa, ya que hay experiencias, como en India, que demuestran el impacto positivo en los estándares de vida, en general, mejorando indicadores de bienestar de la población local (Kshitij, 2015).

En América Latina, una estrategia considerada en el desarrollo forestal comunitario (DFC) y en las EFC ha sido el acompañamiento externo a la comunidad (Bonita et al., 2002; Sabogal et al., 2008). En este ámbito latinoamericano, México y algunos países de América Central son los más avanzados en la promoción del DFC y las EFC. Adicionalmente, prevalecen figuras jurídicas para el DFC basadas en el otorgamiento de derechos usufructuarios; sin embargo, los derechos de propiedad comunal se dan en pocos casos, como en los ejidos en México y Guatemala (Dietmar y Donovan, 2008).

Por otra parte, la firma del Tratado de Libre Comercio de América del Norte (TLCAN) ha sido un paso histórico en el proceso de globalización, toda vez que es necesario eliminar barreras comerciales y mejorar las políticas nacionales que favorezcan el comercio (Klooster, 2003; Villarreal, 2010; Secretaría de Economía, 2013). En lo que respecta al desarrollo forestal en México, en particular los ejidos forestales han sido afectados; entre otras causas, por la apertura comercial y la falta de capacidad para competir con mercados extranjeros, factores que impactan directamente en el bienestar de los ejidatarios forestales. 


\section{Antecedentes del desarrollo forestal comunitario sustentable}

\section{Nivel nacional}

Durante el período 2004-2013 la producción forestal maderable ha disminuido de forma constante, iniciando con 6.7 millones de metros cúbicos rollo $\left(\mathrm{m}^{3} \mathrm{r}\right)$ en 2004 y finalizando este período con 5.9 millones de $\mathrm{m}^{3} \mathrm{r}$. Desde inicios de esta década la disminución ha sido continua, con excepción de los años 2006, 2007 y 2012, en los cuales la producción alcanzó un aumento, alcanzando 6.5, 7.0, y 5.9 millones de $\mathrm{m}^{3} \mathrm{r}$, respectivamente, lo que representa un incremento de $0.9 \%, 7.8 \%$ y $7.4 \%$ con respecto al año anterior. Adicionalmente, en los años 2010 y 2011 se registraron las más bajas producciones durante el período, ya que se obtuvo un volumen de 5.6 y 5.5 millones de $\mathrm{m}^{3} \mathrm{r}$, respectivamente, con una disminución de $3.1 \%$ y $2.2 \%$ con respecto al año anterior. Para 2013, la producción forestal maderable se mantuvo prácticamente estable en en comparación con el año anterior con 5.88 millones de $\mathrm{m}^{3} \mathrm{r}$, lo que significó un decremento de $0.5 \%$ (Semarnat, 2013). A la vez, de acuerdo con Semarnat (2013), los principales productos que se obtuvieron durante el año 2013 fueron: la madera para aserrío (escuadría y durmientes) con $74.9 \%$ de la producción (4.4 millones de $\mathrm{m}^{3} \mathrm{r}$ ), los combustibles (leña y carbón) con $11.3 \%$ (662 $000 \mathrm{~m}^{3} \mathrm{r}$ ) y el restante $13.8 \%\left(813100 \mathrm{~m}^{3} \mathrm{r}\right)$ se destinó a celulósicos, chapa y triplay y postes, pilotes y morillos.

En México, el sector forestal es reconocido por la proporción de propiedad social de sus bosques, ya que $51 \%$ de los bosques están bajo propiedad de los ejidos/comunidades forestales (EyCF) (Cubbage et al., 2013), mismos que son una forma de tenencia comunal de la tierra (Bray y Merino, 2004). Existen 2400 ejidos que aprovechan sus bosques y las áreas forestales del país están habitadas por 11 millones de personas, con 43 etnias con 5 millones de indígenas. Por lo tanto, de acuerdo con Segura (2011), la forestería comunitaria constituye un modelo que avanza en la derrama e impacto de sus beneficios, por lo que México continúa aportando experiencias exitosas a nivel mundial, siendo un referente en el manejo de bosques comunitarios y cambio climático.
Por ello, la política forestal en México ha considerado a los bosques y el agua como prioridad nacional y es así como, en el marco de una nueva política sectorial federal 2013-2018, el Gobierno Federal actual establece específicamente la Estrategia Nacional para el Mejoramiento de la Producción y Productividad Forestal (2013-2018) (Conafor, 2013). Esta estrategia tiene tres componentes: fortalecimiento empresarial comunitario, silvicultura y manejo forestal, así como abasto y transformación. (Semarnat-Conafor, 2013a; 2013b). No obstante, el DFC enfrenta desafíos relacionados, entre otros aspectos, con el desarrollo organizacional, cultura empresarial, reconversión industrial y competitividad (Merino, 2001; Bray et al., 2003; Bray y Merino, 2004). Respecto a la industria de la madera, uno de los principales problemas que enfrenta la producción nacional de madera aserrada es la pérdida de competitividad frente a la madera de importación (Flores et al., 2007).

\section{Forestería comunitaria sustentable en México}

En México, la política forestal considera a los bosques y el agua como prioridad nacional y, de acuerdo con ello, se ha reconocido la importancia de la forestería comunitaria (EyCF), como parte fundamental de la estrategia nacional para transitar al desarrollo forestal sustentable, toda vez que son poseedores de $80 \%$ de la superficie forestal nacional (Conafor, 2001). A pesar de la riqueza biológica y el potencial de sus bosques, $55 \%$ de las aproximadamente 12 millones de personas que viven en estas zonas, se encuentran en extrema pobreza. Menos de $15 \%$ de los bosques en México son manejados y la contribución del sector al PIB es pequeña, con un promedio de solo $1.5 \%$ durante el período comprendido entre 2000 y 2009 (Cubbage et al., 2013).

La forestería comunitaria se entiende como una unidad territorial que incluye recursos naturales, ambientales y humanos, y que presenta fundamentalmente un interés común compartido para la búsqueda de un desarrollo armonizado entre sus componentes. Por ello, reviste gran importancia para el logro del desarrollo forestal sustentable, ya que es un medio para la generación de empleo 
local, bienestar social y de manejo ordenado de los ecosistemas forestales; sin embargo, es una realidad que el desarrollo de esta, se ha visto influenciado por el proceso de apertura comercial, lo cual se considera un reto importante para alcanzar el desarrollo forestal sustentable en México.

Los ejidos y comunidades forestales representan un importante componente en la cadena productiva en el sector forestal, y a la vez constituyen un sistema sociocultural y ecológico clave para la realización de un desarrollo de base con una visión de sustentabilidad. Actualmente, entre 7831 y 9047 ejidos y comunidades son dueños de casi $80 \%$ de la superficie forestal de México; por lo que dos condiciones sociales caracterizan la situación de los bosques de México: la pobreza en que viven la gran mayoría de sus habitantes, y el carácter social de su tenencia. Solo $25 \%$ de las comunidades y ejidos con bosques en aprovechamiento llevan a cabo directamente aprovechamientos forestales, con una contribución a la producción industrial maderera nacional de solo $17 \%$. Las comunidades que integran el restante $75 \%$ aprovechan sus bosques en condiciones de arrendamiento (Conafor, 2001). De tal manera que la forestería comunitaria en México tiene varias décadas con diferentes ejidos y comunidades forestales involucradas en el proceso de desarrollo forestal comunitario, por lo que nuestro país es líder en Latinoamérica y en el mundo en este ámbito (Klooster, 2003).

\section{Empresas Forestales Comunitarias}

Las EFC, enfrentan retos relacionados con su decreciente participación en los mercados de productos forestales debido a limitaciones en su nivel de competitividad y en procesos de certificación que les permitan participar en mejores condiciones en este sentido. A la vez, es importante resaltar que de manera complementaria se ha sugerido que la promoción de los productos forestales no madereros, a través del desarrollo de las micro y pequeñas empresas, podrían formar un punto de entrada importante para la reducción de la pobreza y la seguridad alimentaria, sobre todo en los pueblos de África Central y en general en las comunidades rurales (Tieguhong et al., 2012).
El entorno económico actual en el que se desarrollan las EFC, está caracterizado por la integración de mercados y la competencia con productos estandarizados, provenientes de plantaciones y con bajos costos de producción. Debido a lo anterior, es fundamental la comprensión tanto de la rentabilidad como de la competitividad como factores clave de éxito para las EFC. Específicamente, la relevancia de la competitividad ha ido creciendo, siendo un término que está presente en todos los aspectos que tienen que ver tanto con las políticas públicas de desarrollo como a nivel empresa con las estrategias para su crecimiento y supervivencia.

Lo anterior justifica que el papel del sector social es imprescindible para lograr en el futuro un desarrollo forestal sustentable. La experiencia actual, aun en pocos ejidos y comunidades forestales exitosas, subraya esa conclusión ya que manejan sus bosques para el beneficio local. Sin embargo, en general a pesar de que la tenencia del bosque es comunal, las comunidades y ejidos han sido enajenados de su recurso y no han logrado obtener los beneficios esperados con un enfoque de desarrollo sustentable. A la vez, el desarrollo organizacional con visión empresarial ha constituido un desafío importante para el fortalecimiento de las empresas forestales comunitarias; de tal manera que existen investigaciones realizadas relacionadas con el alcance de una conciencia organizacional para mejora de la condición de desarrollo de la empresa; para ello, algunos estudios han aplicado la metodología denominada "Whole Scale Change (Real Time Strategic Change)" (Arena, 2004), Es una metodología que se aplica para planeación estratégica y cambios de cultura en diseños de trabajo organizacional.

En el país, los principales estados productores forestales en 2013 fueron: Durango (32.80\%), Chihuahua (16.79\%), Michoacán (7.76\%), Oaxaca (7.13\%) y Veracruz $(4.93 \%)$ que contribuyeron con $69.41 \%$ de la producción total, equivalente a 4.1 millones de $\mathrm{m}^{3} \mathrm{r}$ (Semarnat-Conafor, 2004; Semarnat, 2013). Estos estados mencionados, aportan casi $70 \%$ de la producción maderable nacional (Banco Mundial, 1995; Inegi, 2003; Semarnat, 2013). 


\section{Región norte de México: sistema región forestal}

\section{Chihuahua y Durango}

Chihuahua, de acuerdo con la carta de Uso de Suelo y Vegetación Serie III del Inegi (2003), cuenta con una extensión territorial de 24705281.72 ha, de las cuales 22066520 ha se consideran superficie forestal, y estas representan $15.8 \%$ de la superficie forestal nacional, la cual es de 139692886 ha. Para el año 2011, la producción maderable en Chihuahua fue de $1006824 \mathrm{~m}^{3} \mathrm{r}$, representando $18.30 \%$ de la nacional, que es de 5501 $085 \mathrm{~m}^{3} \mathrm{r}$. En el estado destacan tres principales productos: escuadría, celulósicos, chapa y triplay (Gobierno del estado de Chihuahua et al. 2012).

Por otra parte, en Durango existen aproximadamente 500 ejidos y comunidades forestales con cinco millones de hectáreas arboladas, de las cuales casi dos millones se encuentran bajo algún programa de manejo forestal. En ellos, los recursos maderables disponibles son similares a los descritos para el estado de Chihuahua. Además en ambos estados, las actividades forestales económicas principales son el abastecimiento y venta de trocería y la industria de aserrío; adicionalmente, existe una limitada visión empresarial a corto, mediano y largo plazo y limitada competitividad, por ejemplo, existe falta de disposición para integrase horizontal o verticalmente y establecer alianzas con otros ejidos o inclusive con particulares.

En suma, esta región forestal del norte de México se caracteriza por ineficiencia en los procesos de producción, productividad y comercialización, dando como resultado altos costos de producción de la madera y baja competitividad con respecto a los productos de importación.

Es fundamental indicar, para la justificación de la dimensión y alcances del estudio, que los estados de Chihuahua y Durango en conjunto representan cerca de $20 \%$ de la superficie total del país (Inegi, 2011). En consecuencia, es necesario resaltar que los dos estados con mayor producción forestal para el año 2013 fueron Durango y Chihuahua, con una participación conjunta de $49.60 \%$ de la producción forestal maderable total (Semarnat, 2013). Esto es significativo en el nivel de relevancia que representan en su conjunto estas dos entidades desde el punto de vista forestal y en particular del desarrollo forestal comunitario sustentable, considerando una dimensión clave para México como región forestal, y a su vez como clúster forestal en un entorno nacional pero también internacional en el contexto de la globalización económica. Por lo que como resultado del estudio se plantean propuestas de mejora en diferentes aspectos analizados en el mismo.

\section{OBJETIVOS}

Considerando las condiciones del desarrollo forestal comunitario descritas de los estados de Chihuahua y Durango, así como el contexto nacional y de la globalización económica, los objetivos de la investigación fueron:

1. Analizar el desarrollo forestal comunitario sustentable en la región norte de México, particularmente en los estados de Chihuahua y Durango, como un sistema regional forestal-clúster forestal, en relación con: organización comunitaria, flexibilidad, innovación, capacidad de respuesta, competitividad y colaboración y comercialización, ante la continua apertura de mercados globalizados.

2. Establecer una estrategia integral con visión holística para impulsar el desarrollo forestal comunitario sustentable de esta región en el contexto de la globalización económica.

\section{MATERIALES Y MÉTODOS}

\section{Área de estudio}

El estudio se realizó en los estados de Chihuahua y Durango (Fig. 1), considerados en conjunto como un sistema forestal-clúster forestal por sus condiciones similares de EyCF/EFC con bosques de clima templado frío, y por ser los de mayor producción forestal en México.

\section{Sujetos de estudio}

De acuerdo con el nivel de integración de los silvicultores y las categorías establecidas de la tipología de productores forestales se consideró la siguiente para la definición de unidades de estudio (Semarnat-Conafor, 2013b): a) cate- 


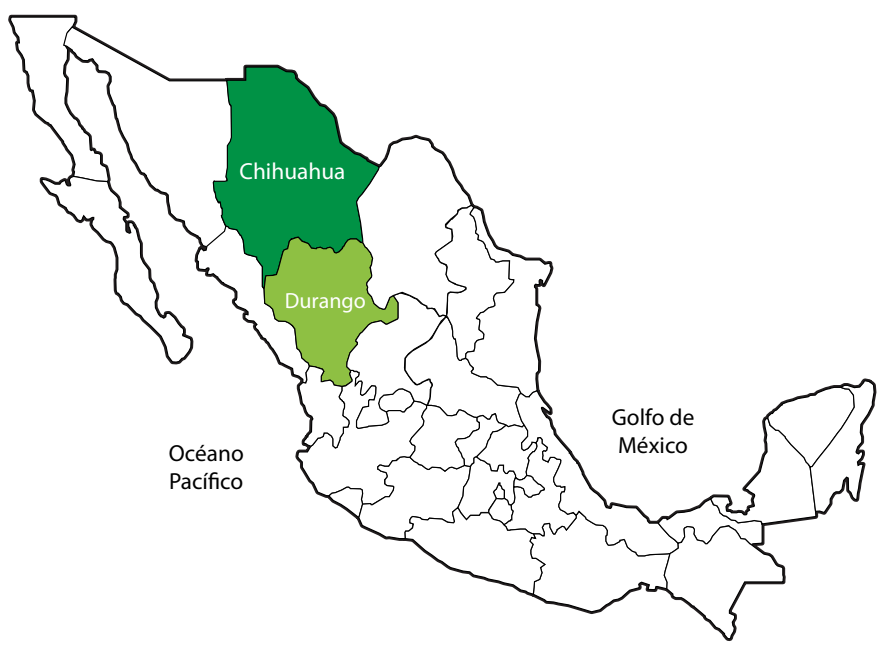

Figura 1. Mapa del área de estudio en: http://paraimprimir. org/mapa-de-la-republica-mexicana-sin-nombre-paraimprimir/. Luján y Vázquez (2013).

goría 1: silvicultores potenciales, siendo aquellos que cuentan con el recurso forestal maderable pero no lo aprovechan; b) categoría 2: silvicultores que venden arbolado en pie, estos poseen recursos forestales que venden a terceras personas que realizan el aprovechamiento; c) categoría 3: silvicultores productores de materias primas forestales, ellos tienen el recurso forestal y lo aprovechan, vendiendo la madera en rollo; y d) categoría 4: silvicultores con capacidad de transformación y comercialización, estos producen madera en rollo y realizan la transformación industrial primaria de la misma y su comercialización.

\section{Variables de estudio}

Se estudiaron las siguientes variables: organización comunitaria, flexibilidad, innovación, capacidad de respuesta, competitividad y colaboración y comercialización. La organización comunitaria considera la estructura organizacional, procedimientos administrativos y funciones laborales de EyCF/EFC; la flexibilidad, está relacionada con la cultura organizacional, nivel de integración productiva, sensibilidad y motivación a los cambios; la capacidad de respuesta e innovación, señala el grado en que el ejido o comunidad tiene un nivel de respuesta oportuna o no a la presencia de cualquier factor relacionado con el desarrollo del mismo; la competitividad y colaboración, se refiere al grado de disponibilidad del ejido o comunidad para colaborar e integrarse con otros ejidos e instituciones para lograr mejor competitividad.

\section{Fuentes de información}

Se consultaron fuentes primarias y secundarias. Las primarias consistieron en entrevistas a informantes clave, incluyendo productores forestales ejidales (silvicultores), prestadores de servicios técnicos forestales y expertos nacionales e internacionales. Como fuentes secundarias se consideraron documentos técnicos y bases de datos, entre otros, relacionados con la temática de estudio.

\section{Unidades de estudio: criterios, tamaño y selección de la muestra}

Para la determinación de la muestra, se utilizaron los criterios siguientes: la tipología de las cuatro categorías de productores indicadas en la sección de "Sujetos de estudio", la superficie forestal de predios, el número de predios bajo aprovechamiento forestal, realizándose la selección al azar. Para ello, se determinó una muestra representativa de 40 EyCF en total, siendo 20 unidades en cada estado (5 ejidos de cada categoría por estado). Adicionalmente, se consideraron representativamente un total de 20 expertos y tomadores de decisión a nivel estado y nacional, incluyendo representantes de instancias como Conafor, Semarnat, organizaciones de productores, organizaciones de industriales, organizaciones no gubernamentales, académicos e investigadores.

\section{Diseño y aplicación de instrumentos}

La entrevista fue el instrumento utilizado para EyCF e informantes clave antes mencionados. Se diseñaron dos cuestionarios: para el caso de los EyCF, el instrumento diseñado incluyó las variables ya indicadas. Además, el otro cuestionario incluyó aspectos de política forestal, estructura organizacional para el desarrollo forestal, entorno internacional para el desarrollo forestal comunitario, investigación y educación forestal, entre otros, como información de referencia contextual. 


\section{Sistematización y análisis de la información}

La información obtenida se procesó en una base de datos diseñada en Excel@, en la cual se capturaron las respuestas obtenidas de las encuestas en los dos estados. Se tuvo una respuesta total de $75 \%$, así como la de los informantes claves con $70 \%$ de respuesta del total. Se analizó la información de los dos estados en conjunto, como un sistema, siendo similar el comportamiento de las variables analizadas en ambos estados y obteniento respuestas homogéneas en su conjunto.

\section{RESULTADOS}

\section{Perfil del Desarrollo forestal comunitario sustentable: Chihuahua y Durango como un sistema regional forestal}

\section{Organización comunitaria, flexibilidad, innovación y}

\section{capacidad de respuesta}

Los EyCF presentaron limitada organización para la producción, productividad y visión empresarial. En relación con la flexibilidad, la resistencia a los cambios ha limitado el desarrollo en la organización comunitaria, administración, y comercialización. Esto no facilita la adaptación rápida a los cambios internos y del entorno. Aun con ello, en las categorías 3 (productores de materias primas forestales) y 4 (transformación industria) se identificaron mayor flexibilidad, capacidad de respuesta e innovación tecnológica, ya que han invertido en mayor grado en sus unidades de producción en capacitación, maquinaria y comercialización.

En los procesos de innovación, los EyCF manifestaron aspiraciones y necesidad de diversificarse e incursionar en nuevos mercados, con nuevos productos o servicios. Sin embargo, es importante considerar otros factores que limitan las oportunidades de desarrollo y los procesos de innovación/diversificación, tales como la falta de organización, recursos económicos, y falta de interés.

\section{Competitividad y colaboración}

La competitividad se ve afectada por altos costos de producción forestal, siendo el transporte el concepto que más impacta por sus altos costos (60\%). En la percepción de los productores forestales, los precios bajos de compra en productos importados son el principal efecto de la apertura de mercados; esto se debe a los precios reducidos de los productos importados en comparación con los locales (Fig. 2).
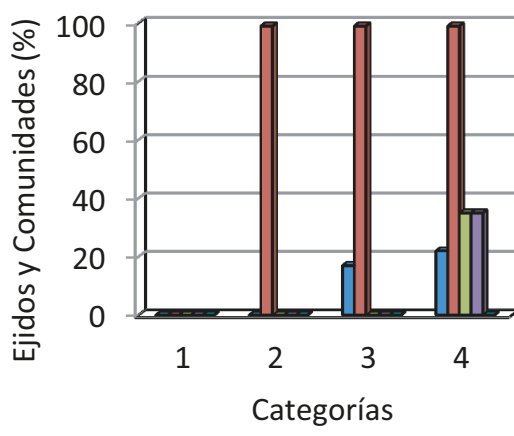

Menor demanda

Precios Bajos

Exigencias de calidad

$\square$ Baja competitividad

$\square$ Otros

Figura 2. Percepción de ejidos y comunidades forestales acerca de los efectos de la apertura comercial y competitividad.

Fuente: Elaboración propia con datos tomados en ejidos forestales en estudio en ambos estados como región forestal.

Adicionalmente, en la figura 3 se observa que para las categorías 3 y 4 (mayor nivel de integración), la calidad es el factor que más afecta la competitividad de productos forestales de EyCF.

En relación con la colaboración, los EyCF reconocen limitada asociatividad y cultura de integración. Por lo tanto, como lo han establecido Linda et al. (1991) y Cubbage et al. (2013), es necesario fomentar e impulsar la asociatividad, considerando la integración tanto horizontal como vertical.

\section{Comercialización de productos forestales}

En la figura 4, se ilustran los procesos de comercialización identificados; tal como se muestra, en los casos más frecuentes (categorías 1, 2 y 3), el proceso se da con la participación de productor-intermediario-consumidor final, que en muchos casos no ofrece las mejores condiciones para el intercambio comercial. A la vez, se observa que en 


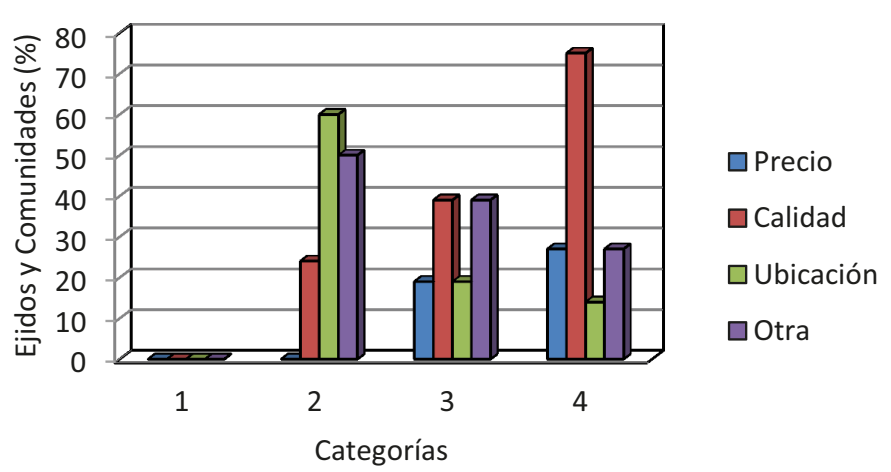

FIgURA 3. Factores que afectan la competitividad de los productos forestales en los ejidos y comunidades forestales.

Fuente: Elaboración propia con datos tomados en ejidos forestales en estudio en ambos estados como región forestal.

la cuarta categoría predomina la participación de productor-mayorista dado su nivel de integración.

Por otra parte, los EyCF no cuentan con estudios de mercado y planes estratégicos para la comercialización de productos forestales; además, la percepción de los productores identifica como principal problema los precios bajos en productos de importación y, en consecuencia, baja demanda de los propios.

\section{DISCUSIÓN}

Como lo indica Messier (2015), es necesario establecer y operar principios amplios y promover cambios que permitan incrementar las capacidades adaptativas de los bosques y el sector forestal con sus diferentes actores ante un futuro de incertidumbre. Ante ello, es evidente que lograr la integración entre los actores de la actividad forestal de una región no es sencillo bajo los paradigmas actuales, caracterizados por un comportamiento individualista y visión a corto plazo de los participantes en cada eslabón de la cadena productiva.

Además, los diversos actores de cada eslabón se perciben a sí mismos como empresas totalmente independientes de los otros eslabones y también con respecto a empresas similares (dentro de cada eslabón); por lo tanto, sus acciones no buscan integrarse en un fin común, sino

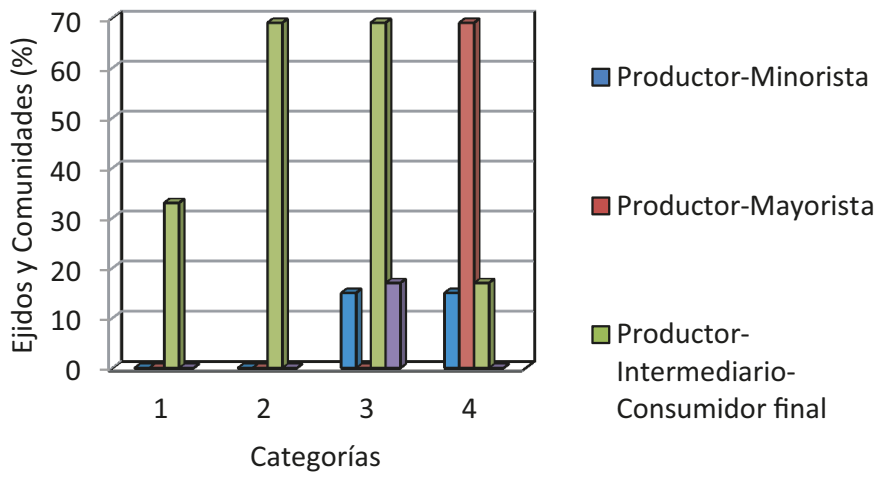

Figura 4. Proceso de comercialización de los ejidos y comunidades forestales según los canales de comercialización.

Fuente: Elaboración propia con datos tomados en ejidos forestales en estudio en ambos estados como región forestal.

que son aisladas, procurando cada una obtener el máximo beneficio (o menor costo) posible, sin tomar en consideración su influencia en el costo o beneficio de los eslabones anteriores o posteriores. Dichos paradigmas no resultan apropiados para lograr que la actividad forestal sea competitiva en el contexto de la globalización económica.

Por ello y considerando la situación prevaleciente en el norte de México en lo relativo al desarrollo forestal comunitario sustentable, los resultados obtenidos en las variables estudiadas arriba citadas en los capítulos de metodología y resultados tienen relación, en general, con lo indicado por Bray y Merino (2004), quienes señalan que el desarrollo forestal comunitario en México enfrenta desafíos relacionados, entre otros aspectos, con el desarrollo organizacional, cultura empresarial, reconversión industrial y competitividad. Por tal motivo, el estudio establece en cada variable propuestas de mejora que contribuyen a impulsar acciones estratégicas que faciliten y atiendan, a través del tiempo, limitantes antes indicadas de dicho desarrollo comunitario, y a la vez el mejoramiento del desarrollo forestal comunitario sustentable en la región norte de México. A continuación se presenta el comportamiento y discusión de cada variable estudiada y su propuesta de mejora. 
En lo referente a la condición de la organización comunitaria como una limitante para el desarrollo forestal comunitario con visión de sustentabilidad, es coincidente con lo referido por Cubbage et al. (2013), ya que menciona que el nivel de organización limita el desarrollo de ejidos y comunidades, debido a que no permite tener la capacidad de respuesta adecuada en aspectos como administración y comercialización con oportunidad, eficiencia, eficacia y efectividad. Esta condición, también coincide con lo establecido en el Programa de Desarrollo Forestal Sustentable del estado de Chihuahua 2012: actualización en el marco del Plan Estatal de Desarrollo 2010-2016 (Gobierno del estado de Chihuahua et al., 2012), en el cual se indica que hay escaso nivel de organización comunitaria en EyCF con visión empresarial. Sin embargo, Antorini (2005) establece que las comunidades con un mayor nivel de integración vertical tienen un campo más amplio de experiencia en organización empresarial y que es una estrategia que se debe de continuar impulsando la aplicación de principios de asociatividad, organización comunitaria y participación democrática, entre otros, en los procesos de toma de decisiones comunitarias. Lo anterior, sustentado en lo que menciona Witek-Crabb (2012), ya que estudios han mostrado que existe una correlación positiva con significancia entre el nivel de sustentabilidad de las prácticas de administración estratégica y desarrollo organizacional en particular y la efectividad en los mercados de las empresas.

En relación a la organización por medio de alianzas y asociaciones, se requiere establecer y operar figuras asociativas con criterio empresarial que permitan establecer y consolidar empresas comunitarias forestales y, por ende, disminuir costos de producción, lograr un mejor balance económico, realizar operaciones directas de sus productos, ser una fuente de autofinanciamiento y comercialización de productos, entre otros. Lo anterior, tomando como base que el desarrollo organizacional con visión empresarial ha constituido un desafío importante para el fortalecimiento de las empresas forestales comunitarias.

Además, es necesario mejorar la administración y organización de los ejidos forestales y sus empresas fores- tales comunitarias, dando capacitación para fortalecer el capital social y humano, así como a los administrativos, quienes muchas veces simplemente son los mismos directivos de los ejidos y carecen de formación técnica o administrativa; también dicha capacitación a personal operador de apoyo a los procesos organizacionales y administrativos.

En relación con la flexibilidad, y debido a la resistencia a los cambios, es necesario considerar lo que indica Improven (2013a), señalando que el nuevo escenario de las organizaciones exige reaccionar de forma rápida a las condiciones internas y externas en constante cambio. A la vez, de acuerdo con Improven (2013b), existen factores clave a considerar para avanzar en los procesos innovativos, como son la creatividad y espíritu innovador para impulsar procesos de mejora continua para lograr organizaciones dinámicas, transversales y colaborativas. Adicionalmente, se requiere fortalecer los procesos de inducción, sensibilización y motivación a los ejidatarios para apoyar e influir en la relevancia de aplicar esta cualidad de la flexibilidad en los procesos de desarrollo comunitario sustentable.

Respecto a competitividad y colaboración, Cubbage et al. (2013) en su estudio de competitividad y acceso a mercados de empresas forestales comunitarias en México, indica que las EFC de México presentan costos de producción relativamente altos en todas las etapas de la cadena productiva. Lo anterior, se atribuye en parte a las grandes distancias a centros de comercialización y a las condiciones deficientes de los caminos y medios de transporte utilizados, lo cual coincide con lo indicado por Indufor (2001). En efecto, en concordancia con Cubbage et al. (2013), y de acuerdo con los resultados obtenidos, los ejidos y comunidades forestales no son competitivos debido a altos costos de producción, bajos niveles de capacitación, deficiente organización, ausencia de planeación y seguimiento en el mediano y largo plazos y la limitada asociatividad que permitan manejar sus empresas con rentabilidad. Por ello, es necesario aplicar estrategias de funcionamiento en la cadena productiva forestal con criterios de eficiencia, eficacia y efectividad que coadyuven al mejoramiento de los niveles de competitividad. De tal forma 
que es importante referir a lo establecido por Trejo (2013), quien indica que la fuerte competencia y la complejidad prevaleciente en momentos en que la globalización tiene cada vez más auge e impacto, están marcando tendencias para que las organizaciones concentren sus esfuerzos en gestionar estratégicamente y de manera participativa sus capacidades para impulsar un desarrollo sustentable con visión de futuro. Además de que la experiencia de las organizaciones indica que el mejor camino para alcanzar la capacidad de competir es a través de la tecnología e innovación, como lo señala Carroz (2005).

Referente a la colaboración, dada la limitada cultura de integración y asociación por parte de los productores, Scherr et al. (2003) y Mota (2002) coinciden con las estrategias marcadas en el Programa Estratégico para México 2025, en el cual se establece que la integración vertical u horizontal, es una alternativa positiva para enfrentar los retos de la apertura comercial. Esto se hace evidente con los esfuerzos que se están realizando en la integración de EyCF, con el apoyo de la Comisión Nacional Forestal, por medio de su programa de Integración de Cadenas Productivas Forestales, con avances específicos importantes en algunas de ellas: Cadena Productiva Forestal Región Madera S.A. de C.V. y Cadena Productiva Tarimas, Muebles y Laminados S.P.R. de R.L. de Durango (Conafor, s/f a). Adicionalmente, en específico, FIRA (2010) ha apoyado iniciativas de integración, como es el caso de la "Integradora Comunal Forestal de Oaxaca S.A de C.V”, integrada por empresas forestales de las comunidades de Pueblos Mancomunados, Ixtlán de Juárez y Santiago Textitlán de Oaxaca. Todas ellas, con indicadores importantes en los avances en el proceso de desarrollo comunitario sustentable.

Por otra parte, sobre comercialización de productos forestales, la percepción de los productores sobre los efectos de la apertura de mercados en el proceso de la comercialización no es favorable, ya que hay precios bajos de los productos extranjeros y la competencia desleal por parte de aprovechamientos clandestinos, entre otros factores, lo que no permite a los productos nacionales ser competitivos en precios, y ello ha ocasionado una menor demanda de los mismos, como lo afirma Chapela (2012), quién explica que México es un importador neto de materias primas y productos manufacturados de madera (Conafor, $\mathrm{s} / \mathrm{f}$ b). Por ejemplo, mientras China es un gran importador neto de productos forestales, México es importador neto de madera de otros países e importador de productos de madera de China (Elizondo, 2005). Sin embargo, es recomendable buscar alternativas comerciales para los diferentes productos forestales para así obtener más provecho del bosque y disminuir los desperdicios. Por ejemplo, algunas empresas forestales comunitarias ya están comercializando el encino como carbón y/o leña, lo que indica la existencia de demanda por estos productos, además de los que tradicionalmente se han comercializado.

En consecuencia, considerando el sistema regional forestal del norte de México (Chihuahua y Durango), se identificaron los siguientes impactos de la globalización económica en el desarrollo forestal comunitario sustentable: bajo nivel de rentabilidad y competitividad de la actividad forestal, disminución del empleo, cultura organizacional y administrativa tradicional y poco flexible, así como necesidad de avanzar en la certificación forestal.

Por consiguiente, se enfatiza la necesidad de aplicar la silvicultura intensiva para el mejoramiento de los índices de producción y productividad de los bosques; modernización de la tecnología para los procesos de aprovechamiento y transformación; fortalecimiento de los procesos de planeación estratégica, acompañamiento multidimensional, seguimiento y evaluación para elevar los niveles de eficiencia, eficacia y efectividad; impulsar la realización de estudios de mercado para la identificación de nichos que sirvan de base para orientar el tipo de productos que deben de ser elaborados; fortalecer los procesos organizacionales entre productores, industriales y comercializadores que permitan potenciar esfuerzos que se traduzcan en impactos positivos en el desarrollo del sector forestal; implementar programas de capacitación continua para el fortalecimiento del capital humano y social, así como los niveles organizacionales del sector; e implementar esquemas de financiamiento accesibles que impulsen el desarrollo del sector. Para ello, a continuación se plantea una estrategia 
integral que permita en el tiempo impulsar el desarrollo forestal comunitario sustentable en la región de estudio.

\section{Estrategia integral propuesta para impulsar el desarrollo forestal comunitario sustentable}

Factores como el creciente interés global por la protección y aprovechamiento de los recursos naturales, la importancia que van ganando los bosques naturales como elementos importantes para promover cada vez más el desarrollo forestal comunitario sustentable, así como la mitigación y adaptación al cambio climático, ha, abierto la posibilidad de nuevos mercados que pueden ser una fuente de ingreso adicional para los ejidos; por ejemplo los "bonos de carbono" que se pagan por el carbono acumulado en el bosque y/o pagos que se hacen por la conservación del agua, a través de la protección de los bosques (Cubbage et al., 2013).

Por ello, considerando los impactos de la globalización económica ya mencionados y las variables estudiadas, se establece la propuesta de una estrategia integral participativa sustentada en una visión sistémica, tomando como base el proceso de integración horizontal/vertical. Se sugiere que esta estrategia incluya tres grandes apartados: a) la estrategia básica de integración como clúster forestal y el principio de asociatividad, b) el establecimiento de la vinculación con el empoderamiento de ejidos y comunidades forestales y c) la consideración del contexto de la globalización económica.

En relación con el clúster forestal-asociatividad, esta estrategia integral con visión sistémica promueve un mayor nivel de competitividad y desarrollo socioeconómico, privilegiando el manejo forestal sustentable, y otros beneficios potenciales como son: oportunidades de empleos, sustitución de importaciones, oportunidades de exportación, diversificación de productos, penetración en nuevas ventanas, reducción de costos de producción, incremento en ventas, nuevo conocimiento y experiencia, mayor poder de autogestión, y acceso a nuevas tecnologías, entre otros. Lo anterior, en función de que las EFC en México venden a mercados nacionales y locales en los que la demanda es alta así como los precios y los productos se venden en casi su totalidad. Los competidores internacionales, sin embargo, representan una amenaza para la sostenibilidad financiera las EFC, ya que producen a costos más bajos que las EFC mexicanas, quienes deben prepararse para una posible competencia internacional.

En apoyo a lo anterior, será recomendable que las empresas forestales comunitarias reinviertan una porción de sus ganancias para mantener la maquinaria y los activos de la empresa, que en muchos casos es precaria o está en una situación de grave desgaste.

Para el caso del empoderamiento de ECF, la estrategia propuesta integra la necesidad de considerar un conjunto de factores resultantes de ese empoderamiento, como son: recursos económicos, capital social, tecnología, autogestión, organización y bienestar social. Estos factores deben interactuar con un criterio de efecto multiplicador que potencie la capacidad de respuesta de la forestería comunitaria en su conjunto. Lo anterior, se reflejará en su nivel de competitividad que les permita mayor participación con éxito en los mercados nacionales e internacionales.

Respecto al contexto de la globalización económica, la fase de la integración del clúster forestal y el empoderamiento de ejidos forestales deben enlazarse al mundo de la apertura comercial a través de ventajas competitivas sostenibles. Para ello, existen en el contexto internacional ventanas de mercado, como lo señala De la Mora (2003) en su estudio el Comercio Internacional y el Sector Forestal en México, mismas que representan oportunidades para el desarrollo de la forestería comunitaria con criterios de productividad, competitividad y sustentabilidad ambiental.

En consecuencia, para el éxito de esta estrategia de integración se deben de considerar, entre otras, las siguientes acciones: cambio de paradigmas, apoyos gubernamentales, educación, capacitación, y organización de alianzas y asociaciones. En referencia al cambio de paradigmas, en primer lugar hay que cambiar los que tradicionalmente han predominado en la actividad forestal en México, y adoptar otros mejores, que sean funcionales en el nuevo contexto. En algunos casos los paradigmas a adoptar pueden surgir de la adaptación de experiencias de otros paí- 
ses, como puede ser adquirir y operar en forma común, mediante esquemas de cooperación y administración adecuados, la maquinaria y equipos de extracción e industrialización necesarios para procesar la madera de varios EyCF y de otros pequeños propietarios.

La estrategia integral incluye de manera relevante la educación y capacitación, como acciones básicas para lograr niveles de vanguardia y fomentar una capacitación sistemática y continua con un plan de largo plazo. Por último, se propone aprovechar los programas de apoyo gubernamental para fomentar la integración horizontal y vertical, y fortalecer el período de acompañamiento brindando asistencia técnica, económica y administrativa, a través de una red de asesores especializados y certificados que coadyuven a la integración en ambos sentidos para lograr que el clúster forestal sea competitivo.

Por lo anterior, se plantea la integración horizontal/ vertical que permita potenciar las capacidades e intereses que se encuentre que son comunes entre sectores, para de esa manera facilitar el tránsito a procesos eficientes de comercialización en el contexto de la apertura comercial; además de otros posibles beneficios como: nuevas oportunidades de empleos, sustitución de importaciones, oportunidades de exportación, diversificación a nuevos productos, protección de los mercados actuales y penetración en nuevas ventanas, reducción de costos de producción, incremento en ventas, nuevo conocimiento y experiencia, mayor poder de autogestión y acceso a nuevas tecnologías, entre otros.

\section{Intra e interconectividad de los componentes de la estrategia integral}

La estrategia integral propuesta incorpora la necesidad de que sus tres componentes sean abordados con visión holística: integrar el clúster forestal, lograr el empoderamiento de ECF e incidir mejor en los mercados en el contexto de la globalización económica. Para ello, estos tres componentes deben concebirse intra e interconectados, a través de los mecanismos que constituyen el flujo de producción y comercialización de productos forestales maderables y no maderables, y su respectivo proceso de retroalimentación. En su conjunto, estos mecanismos llevarán a la estra- tegia integral a un proceso de adaptación que permitirá lograr, a través del tiempo, la consistencia y dinámica propia que se requiere para que la forestería comunitaria avance hacia un mayor empoderamiento y competitividad con visión de sustentabilidad.

\section{CONCLUSIONES}

- La globalización económica es irreversible y el desarrollo forestal comunitario sustentable en Chihuahua y Durango no puede excluirse de este proceso, por los efectos ya indicados de esta sobre EyCF/EFC.

- Se identificaron en EyCF/EFC impactos no favorables en rentabilidad, competitividad, cultura administrativa y organizacional y certificación forestal.

- Es necesario impulsar el mejoramiento de los índices de producción y productividad de los bosques; modernizar la tecnología para los procesos de aprovechamiento y transformación; fortalecer los procesos de planeación estratégica participativa, acompañamiento multidimensional, seguimiento y evaluación para elevar los niveles de eficiencia, eficacia y efectividad; impulsar la realización de estudios de mercado para la identificación de nichos que sirvan de base para orientar el tipo de productos que deben de ser elaborados; y fortalecer los procesos organizacionales entre productores, industriales y comercializadores que permitan potenciar esfuerzos que se traduzcan en impactos en el desarrollo del sector forestal.

- Desafortunadamente, los ejidos por sí mismos no pueden lograr la sustentabilidad y capacidad de respuesta a la dinámica del entorno, siendo necesario impulsar en particular el acompañamiento multidimensional (técnico, organizacional, administrativo, financiero, tecnológico, de mercados y del ambiente).

- Es necesario promover cambios de paradigmas y bondades de la asociatividad en EyCF para efectos de hacer frente a la globalización.

- Los productores forestales deben conseguir mayores niveles de integración horizontal y vertical y mayor competitividad, adoptando una lógica organizativa empresarial, y alianzas entre comunidades y empresas. 
Por otra parte, considerando que una de las principales limitaciones en el funcionamiento de EyCF fue la escasa aplicación de sistemas de control y seguimiento con información sistematizada en EyCF/EFC relacionada con sus procesos de desarrollo, es necesario impulsar sistemas de control y seguimiento en EyCF/EFC para lograr mejor toma de decisiones, de forma oportuna y con mayor sustento. Además, de acuerdo con los resultados obtenidos, la actividad que más costos representa comparado con sus ganancias, es el aprovechamiento forestal, por lo que las EFC deberían de ser dueñas de toda la cadena incluyendo el aserradero, y promover un desarrollo integral de procesos.

De la misma manera, es relevante continuar realizando estudios estructurales y operacionales, relacionados con el desarrollo forestal comunitario sustentable en el contexto de la globalización económica, que coadyuven al fortalecimiento de esta estrategia integral participativa con visión holística propuesta. Además, realizar dichos estudios a un nivel más amplio y aplicado en México a nivel regional, estatal y local en el largo plazo. Adicionalmente, existe la necesidad de promover en el futuro mayor integración de la cadena productiva forestal, incluyendo el establecimiento y consolidación de clústeres, alianzas o uniones de ejidos forestales, a través de la promoción de figuras asociativas haciendo uso de convenios.

\section{REFERENCIAS}

Antinori, C. 2005. Vertical integration in the community forestry enterprises of Oaxaca. In: D. Bray, L. Merino-Pérez, and D. Barry, eds. The community forests of México: managing for sustainable landscapes. University of Texas Press. Austin. 241 p.

Arena, M. J. 2004. Enhancing organizational awareness: An analysis of whole scale change. Organization Development Journal 22(1):9-20.

Banco Mundial. 1995. Estudio de revisión del sector forestal y conservación de recursos. división de operaciones de recursos naturales y pobreza rural. Oficina Regional de América Latina y El Caribe. 159 p.

Bonita, M., F. Correa, P. Veijalainen y H. Ahveninen. 2002. Forest clusters: a competitive model for Latin America.
Inter-American Development Bank, Sustainable Development Department. Environment Division. Washington, D.C. Disponible en línea: http://www.iadb.org/sds/doc/ ENV-IDBForestClústers.pdf.

Bray, D., L. Merino-Pérez, P. Negreros-Castillo, G. SeguraWarnholtz, J.M. Torres-Rojo y H. Vester. 2003. Mexico's community-managed forests: A global model for sustainable landscapes?. Conservation Biology 17(3):672-677.

Bray, D. y L. Merino-Pérez. 2004. Los bosques comunitarios de México: logros y desafíos. Editora Infagón, México.

Carroz, U.D. 2005. Modelo de gestión estratégica para el desarrollo de capacidades tecnológicas. Compendium 8(15):5-19.

Chapela, G. 2012. Problemas y oportunidades en el mercado para las empresas sociales en México. In: USAID-Abt Inc. Consejo Civil Mexicano para la Silvicultura Sostenible y Universidad Autónoma Chapingo, eds. Competitividad de las empresas sociales forestales en México (en proceso de impresión). Editorial Universidad Autónoma Chapingo.

Conafor (Comisión Nacional Forestal). 2001. Programa estratégico forestal para México, 2025. Disponible en línea: http:// www.conafor.gob.mx

Conafor (Comisión Nacional Forestal). S/F a. Programa para la integración de cadenas productivas. Guadalajara, Jal., Mex.

Conafor (Comisión Nacional Forestal). 2013. Estrategia de incremento a la producción y productividad forestal nacional 2013-2018. Guadalajara, Jal., Mex.

Conafor (Comisión Nacional Forestal). S/F b. Diagnóstico del Comercio Internacional Forestal de México. Guadalajara, Jal. $405 \mathrm{p}$.

Cortave, M. 2003. La experiencia de ACOFOP en Petén, Guatemala: Un proceso arduo de gestión política. Centro de Derecho Ambiental y de los Recursos Naturales/Coordinadora Indígena-Campesina de Agroforestría Comunitaria, San José, Costa Rica.

Cubbage, F., R. Davis, D. Rodríguez, G. Frey, R. Mollenhauer, Y. Elsin, I. González-Hernández, H. Albarrán, A. Mercedes y D. Chemor. 2013. Competitividad y acceso a mercados de empresas forestales comunitarias en México. Profor, Conafor, Banco Mundial. México. 132p. 
De La Mora G. 2003. Comercio internacional y el sector forestal en México (balanza). Disponible en línea: http://www. ccmss.org.mx/modulos/biblioteca_consultar.php?folio=25

Dietmar, S. y J. Donovan. 2008. Capacidades empresariales para el desarrollo de empresas forestales comunitarias. In: C. Sabogal, W. De Jong, B. Pokorny y B. Louman, eds. Manejo forestal comunitario en América Latina. Experiencias, lecciones aprendidas y retos para el futuro. Bogor, Indonesia: Centro para la Investigación Forestal (CIFOR).

Elizondo, A. 2005. El mercado de la madera en México. Estudio para recomendaciones de política para expandir el mercado de madera certificada. Informe Final. México: Instituto Nacional de Ecología. Disponible en: http://www. ine.gob.mx/descargas/dgipea/maderas_02_elizondo_ study.pdf.

FIRA (Fideicomisos Instituidos en Relación con la Agricultura). 2010. 200 Casos de éxito FIRA en el sector rural mexicano. México.

Flores, R., E. Serrano, V. Palacio y G. Chapela. 2007. Análisis de la industria de la madera aserrada en México. Madera y Bosques 13(1):47-59.

Friedmann, J. 1992. Empowerment: The politics of alternative development. Blackwell. Cambridge, MA.

Gill, L. 2002. The first nations reclaim a temperate rain forest. In: Ford Foundation, eds. Sustainable solutions: Building assets for empowerment and sustainable development. Nueva York.

Gobierno del Estado de Chihuahua, C. Luján, M. Olivas y S. Vázquez. 2012. Programa de Desarrollo Forestal Sustentable del Estado de Chihuahua. Chihuahua, Chihuahua. $135 \mathrm{p}$.

Hirschman, A. 1993. Getting ahead collectively: grassroots experiences in Latin America. Fundación Interamericana, Arlington, VA.

Improven. 2013a. Organizaciones flexibles, la clave para tener éxito. Newsletter: Allied Consultants Europe-Improven. Disponible en línea: http://www.improven.com/organizaciones-flexibles-la-clave-para-tener-exito/.

Improven. 2013b. ¿Existen silos de gestión en tu organización?. Newsletter: Allied Consultants Europe-Improven. Dispo- nible en: http://improven.com/blog/existen-silos-de-gestion-en-tu-organizacion-2/\#more-348.

Klooster, D. 2003. Campesinos and Mexican forest policy during the twentieth century. Latin American Research Review 38(2):94-126.

Kshitij, C. 2015. The effect of political decentralization and affirmative action on Multidimensional Poverty Index: evidence from Indian States. Journal of Social and Economic Development 17(1):27-49.

Indufor. 2001. Diagnóstico nacional del sector forestal. Versión

\subsection{Helsinki, Finlandia.}

Inegi (Instituto Nacional de Estadística y Geografía). 2011. Panorama Sociodemográfico de México. México, 104 p.

Inegi (Instituto Nacional de Estadística y Geografía). 2003. Anuario de estadística por entidad federativa. Aguascalientes, Ags., México. 660 p.

Linda, H., J. Markusen y T. Rutherford. 1991. Trade liberalization in a multinational-dominated industry: A theoretical and applied general-equilibrium analysis. NBER Working Papers 3679, National Bureau of Economic Research, Inc.

Luján, C. y S. Vázquez. 2013. Mapa del área de estudio. Disponible en línea: http://paraimprimir.org/mapa-de-la-republica-mexicana-sin-nombre-para-imprimir/.

Merino, L. 2001. Las políticas forestales y de conservación y sus impactos sobre las comunidades forestales. $41 \mathrm{p}$.

Messier, C., K. Puettmann, R. Chazdon, K.P. Andersson, V.A. Angers, L. Brotons, E. Filotas, R. Tittler, L. Parrott, L. y S.A. Levin. 2015. From management to stewardship: viewing forests as complex adaptive systems in an uncertain world. Conservation Letters.

Mota, J.L. 2002. Estudio de caso de integración horizontal: Asociación de productores de reposición forestal y comercializadores de carbón del municipio de Nagarote (ecocarbón) en Nicaragua. Banco Interamericano de Desarrollo (BID).

Nolan, M. 2001. Community based forest management: Commercial harvesting of the rainforest of Indonesia. The International Forestry Review 3(3):231-235.

Porter, M. 1990. The competitive advantage of nations. Mac Millan. Londres. 
Redclift, M. 1987. Sustainable development: exploring the contradictions. Methuen. Nueva York.

Sabogal, C., W. de Jong, B. Pokorny y B. Louman. 2008. Manejo forestal comunitario en América Latina. Experiencias, lecciones aprendidas y retos para el futuro. Indonesia: Cifor, Bogor.

Scherr, S., A. White y D. Kaimowitz. 2003. A new agenda for conservation and poverty reduction: Making forest markets work for low-income producers. Forest Trends. Center for International Forestry Research. Washington, DC.

Secretaría de Economía. 2013. Tratados y acuerdos firmados por México. Disponible en línea: http://www.economia. gob.mx/comunidad-negocios/comercio-exterior/tlcacuerdos.

Segura, G. 2011. La visión del sector desde la perspectiva académica, del gobierno federal yde organismos internacionales. In: Comisión Nacional Forestal, eds. Encuentro nacional de silviculura comunitaria. Memoria del evento. México, D.F.

Semarnat (Secretaría de Medio Ambiente y Recursos Naturales) - Conafor (Comisión Nacional Forestal). 2004. Anuario estadístico de la producción forestal. Disponible en línea: http://148.223.105.188:2222/snif_portal/index. php?option=com_content $\&$ task=view $\&$ id $=33 \&$ Itemid $=36$.

Semarnat (Secretaría del Medio Ambiente y Recursos Naturales). 2013. Anuario estadístico de la producción forestal. Dirección General de Gestión Forestal y de Suelos. México.

Semarnat (Secretaría del Medio Ambiente y Recursos Naturales) - Conafor (Comisión Nacional Forestal). 2013a. Propuesta de actualización programa estratégico forestal para México 2025, V.4. México, D.F. 158p.
Semarnat (Secretaría del Medio Ambiente y Recursos Naturales) - Conafor (Comisión Nacional Forestal). 2013b. Reglas de operación del Programa Nacional Forestal 2013. Diario Oficial de la Federación. Viernes 8 de marzo de 2013. Secretaría de Gobernación, México.

Smith, B. y V. Cossio. 2008. Competitiveness of Forest Products at Global Markets; with Particular Emphasis on Tropical Forest Products and on Small and Medium Scale Producers. Market Review in the U.S. of Selected Timber, FAO. Disponible en: http://www.fao.org/forestry/media/15024/1/0/.

Villarreal, M.A. 2010. NAFTA and the Mexican economy. Federation of American Scientists Congressional Research Service. RL34733. EUA.

Tieguhong, J.C., O. Ndoye, S. Grouwels, W.A. Mala y J.L. Betti. 2012. Rural enterprise development for poverty alleviation based on non-wood forest products in Central Africa. International Forestry Review 14(3):363-379.

Trejo, N.A. 2013. Desafíos de la competitividad en la frontera norte de México en el marco del reajuste global. (in Spanish). Frontera Norte 25(50):213-221.

Witek-Crabb, A. 2012. Sustainable strategic management and market effectiveness of enterprises. Procedia - Social and Behavioral Sciences 58:899-905.

Manuscrito recibido el 18 de febrero de 2014. Aceptado el 23 de agosto de 2015.

Este documento se debe citar como: Luján Á., C., J.M. Olivas G., H.G. González H., S. Vázquez Á., J.C. Hernández D. y H. Luján Á. 2016. Desarrollo forestal comunitario sustentable en la región norte de México y su desafío en el contexto de la globalización. Madera y Bosques 22(1):37-51. 